\title{
Reliability of an isometric and isokinetic strength testing protocol of the knee and ankle in young adults
}

\author{
S. Gobbo ${ }^{1}$, M. Bergamin 1 , V. Bullo1, M. Bergamo1, D. S. Bocalini', A. di Blasio ${ }^{3}$, L. \\ Cugusi $^{4}$, E. Roma ${ }^{1}$, F. Battista ${ }^{1}$, C. L. Alberton ${ }^{5}$, D.I Neunhaeuserer ${ }^{1}$, A. Frizziero ${ }^{6}$, \\ B. Vendramin ${ }^{1}$, F. Duregon ${ }^{1}$, A. Ermolao ${ }^{1}$
}

1 Sport and Exercise Medicine Division, Department of Medicine, University of Padova, Italy

2 Laboratorio de Fisiologia e Bioquimica Experimental, Centro de Educacao Fisica e Deportos, Universidade Federal do Espirito Santo (UFES), Vitoria, Brasil

3 Department of Medicine and Sciences of Aging, G. d'Annunzio University of Chieti-Pescara, Italy

${ }^{4}$ Department of Medical Sciences, University of Cagliari, Italy

${ }^{5}$ Escola Superior de Educação Física, Universidade Federal de Pelotas, Brasil

6 Department of Neuroscience, University, University of Padova, Italy

\section{CORRESPONDING AUTHOR:}

Marco Bergamin

Sport and Exercise Medicine Division,

Department of Medicine,

University of Padova.

Via Giustiniani, 2

35128 Padova (Italy)

Phone: +39049 8214429

E-mail: marco.bergamin@unipd.it

DOI:

10.32098/mltj.03.2019.08

LEVEL OF EVIDENCE: 2B

\begin{abstract}
SUMMARY
Background: Isokinetic dynamometers are becoming very common in assessing muscle strength and pathology, both in research and clinical practice, but for most of those devices reliability studies are still needed to support their extended use. The aim of this study is to assess the test-retest reliability also in health adults.

Methods: Thirty adults (13 male and 17 females; mean age $25.4 \pm 2.7$ years) were recruited among University students. They participated to two testing sessions ( 7 day apart) in which they performed isokinetic and isometric strength assessment of the knee and ankle flexion and extension.

Results: All variable showed an Intra-class correlation coefficient higher than 0.7 (isometric knee extension 0.96; isokinetic knee extension 0.96; isokinetic knee flexion 0.97; isometric ankle right flexion $\mathrm{pl}$ and flexion do 0.75-0.96; isometric ankle left flexion $\mathrm{pl}$ and flexion do 0.78-0.97; isokinetic ankle right flexion $\mathrm{pl}$ and flexion do 0.88-0.73; isokinetic ankle right flexion $\mathrm{pl}$ and flexion do $0.88-0.85$ ) and paired-sample t-test showed no significant difference. Moreover, most of the recorded values were included within the upper and lower limits of agreement.

Conclusion: Multi-joint evaluation system is a reliable device to assess knee and ankle isokinetic and isometric strength among healthy adults.
\end{abstract}

\section{KEY WORDS}

Isokinetic test; Isometric test; Isokinetic dynamometers; Kneelankle strength; Torque

\section{BACKGROUND}

In the last three decades, many isokinetic devices were developed and become commercially available. Isokinetic devices provide constant velocity with accommodating resistance throughout a joints' range of motion (ROM). For this reason, in both clinical and research settings this type of muscle contraction has become very common in assessing muscle strength and pathology (1). Recently, these measures are used to interpret dynamic muscle function (2). In fact, isokinetic dynamometers provide quantitative information of various muscle groups, including peak torque and the angle at which it occurs, torque at any angle of ROM and the average power of the contraction. Generally, isokinetic devices are used in rehabilitation to assess risk factors 
such as knee and hamstring injuries or imbalances (3), but lately those systems are employed in sport medicine units to evaluate patients' strength for exercise prescriptions, for example the strength of the soleus which could provide a surrogate measure of functional capacity in patients with heart failure (4). Moreover, another use of these devices is in the preseason screening of unilateral and bilateral strength in healthy athletes to avoid injuries during training or challenge (5). In fact, the bilateral muscle asymmetry and muscle imbalances about the knee have been stated as the etiology of many injuries, especially hamstring strains (3). The usefulness of an isokinetic dynamometer depends upon the reproducibility, or reliability, of the equipment, the test protocol and the measurements obtained (6). For these reasons, using a reliable testing technique and instrument it is essential to delineate disability cut-off score and it is fundamental to avoid mistakes in strength and power evaluations of the lower limb.

In this context, several investigations have evaluated the inter-reliability of knee muscles strength indices using different isokinetic dynamometers such as Biodex and Cybex (7). Moreover, other studies have previously evaluated the reliability of isokinetic devices using different angular velocities (8), testing the strength indices of different joints (9) and using isokinetic device to assess isometric strength (10). So many isokinetic devices have been already analyzed, such as Cybex (11), Kin Com (1), iSAM 9000 (12) Biodex System 4 (13).

The reliability of multi-joint evaluation system Prima Plus ${ }^{\circledR}$ (Easytech Srl, Borgo San Lorenzo FI, Italy) was demonstrated in elderly subjects (14). Nevertheless, at the best of authors' knowledge, no studies have assessed the reliability of this device in healthy young adults. Therefore, the aim of this investigation is to assess the test-retest reliability of knee and ankle isokinetic and isometric strength testing protocol in healthy young adults, using a new and untested isometric and isokinetic multi-joint system dynamometer.

\section{MATERIAL AND METHODS}

\section{Participants}

Thirteen male $(25.62 \pm 2.95$ years, height $1.81 \pm 0.06$ meters, weight $74.12 \pm 9.87 \mathrm{~kg}$, BMI $22.63 \pm 2.38)$ and seventeen females (25.24 \pm 2.56 years, height $1.7 \pm 0.05$ meters, weight $60.76 \pm 8.79 \mathrm{~kg}$, BMI $20.98 \pm 2.47)$ young adults recruited among University students agreed to participate in this study. Sample size calculation was based in according to other investigations examining dynamometer measures in young adults $(1,15)$. To this end the following equation was applied $\left.\mathrm{N}=\left(2\left(\mathrm{SD}^{2}\right)\right) *(\mathrm{Z} \alpha+\mathrm{Z} \beta)^{2}\right) / \Delta^{2}$. Assuming $10 \mathrm{Nm}$ as
Table I. Participant characteristics.

\begin{tabular}{lllll}
\hline N. Subjects & $\begin{array}{l}\text { Weight } \\
(\mathbf{k g})\end{array}$ & $\begin{array}{l}\text { Height } \\
(\mathbf{m})\end{array}$ & $\begin{array}{l}\text { BMI } \\
(\mathbf{k g} / \mathbf{m} 2)\end{array}$ & $\begin{array}{l}\text { Age } \\
\text { (years) }\end{array}$ \\
\hline $30(13 \mathrm{M}$, & $67.71 \pm$ & $1.75 \pm$ & $21.69 \pm 2.53$ & $25.4 \pm$ \\
$17 \mathrm{~F})$ & 11.49 & 0.82 & & 2.7 \\
\hline
\end{tabular}

Abbreviation: BMI, body mass index.

significant different, and 20 as an acceptable standard deviation $(\mathrm{SD})$, the equation reported 31 subjects.

Voluntary subjects (age $\geq 20$ y.o.) were recruited from university students. After a general medical examination questionnaire, subject with history of cardiovascular, pulmonary, neurologic, musculoskeletal, or other major systemic problems were immediately excluded.

The experimental purpose and procedure were explained to each participant, and a written consent for their participation in the trial was gave. In addition, participants were administered the Mini Mental State Examination (MMSE) (16), that was used as a screening device to rule out significant cognitive impairments (17). The Ethics Committee of the University of Padova approved all the procedures.

\section{Device}

The multi-joint evaluation system (Prima Plus, Easytech, Italy) is composed by a sit and a mechanic unit. Dimensions are $70 \times 140 \times 135 \mathrm{~cm}$ with an overall weight of $193 \mathrm{~kg}$. The device is conformed to the EU standards for the safety (directive 93/42 CEE). Certificate No. 114091-2012-CE-ITA-NA attests the quality for design, production and final product inspection/testing. The system allowed the evaluation of squat movement, intra- and extra-rotation of the shoulder, knee flexion and extension, and dorsal and plantar flexion of the ankle. The calibration of the dynamometer was performed in 15 different angles (from $0^{\circ}$ to $240^{\circ}$ with $15^{\circ}$ for each one), applying a known weight in a specific point of the lever (Figure 1). Largest error during isokinetic testing is $0.6 \mathrm{Nm}(0.23 \%)$.

\section{Procedure}

The same two investigators performed strength tests in two separate moments (test $\&$ re-test) with an interval period of 7 days between the sessions (18). Before testing, to avoid any learning effects which were frequently associated with strength testing procedures particularly in naive subjects, a separate practice session was scheduled to make participants familiar with the testing procedures. Furthermore, a 


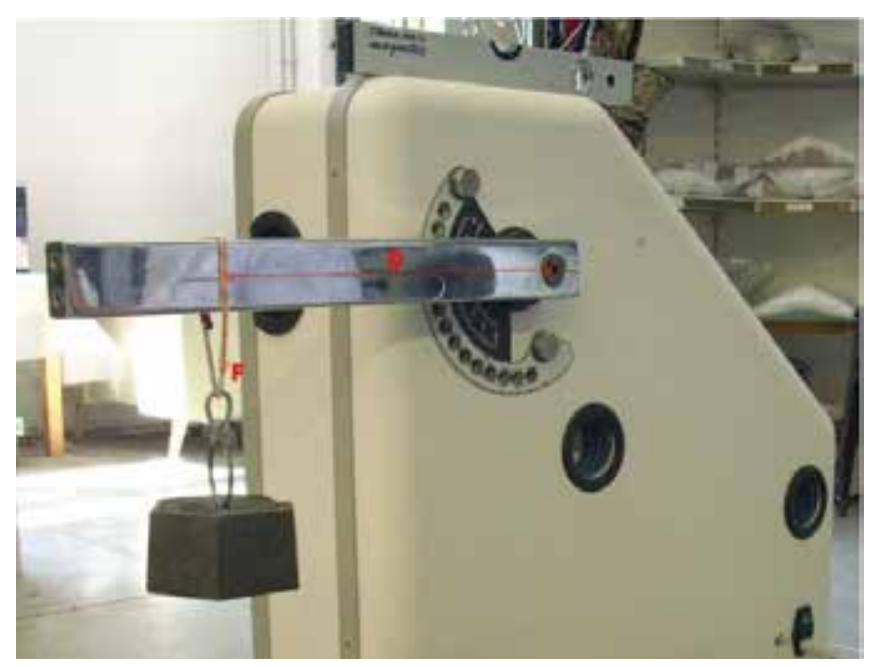

Figure 1. Calibration process, $D$ is the distance between fulcrum and Force (F) exerted to the lever.

warm up session was always performed. A 60-seconds recovery period was allowed between all testing procedures. No verbal encouragements or visual feedback were given and subjects were asked to wear the same shoes and comfortable clothes in all sessions.

The position of the subjects on the multi-joint system was seated with the backrest angled at $90^{\circ}$ to the seat, with their arms across the chest. To minimize body movement and to optimally isolate the movement of knee joints and ankles, specific belts were placed across the thighs, the pelvis, and the shoulders.

Eligible parameters were maximal isometric bilateral knee extension at $75^{\circ}$ of extension, maximal isokinetic bilateral knee extension and flexion with a range of movement between $0^{\circ}$ (anatomic $0^{\circ}$ ) to $85^{\circ}$ of knee flexion, right and left maximal isometric ankle plantar and dorsal flexor at $30^{\circ}$ of plantar flexion and right and left maximal isokinetic ankle plantar and dorsal flexor with a range of movement between $0^{\circ}$ (anatomic $0^{\circ}$ ) to $65^{\circ}$ of ankle plantar flexion. The order of tasks was randomized trying to reduce possible interference effect among them.

During the two knee trials the fulcrum was aligned with the lateral femoral epicondyle, and the shin pad placed $2 \mathrm{~cm}$ above the medial malleoli. Lever arm was set at $75^{\circ}$ extensions, calculated on the maximum knee extension of each participant during maximal isometric bilateral knee extension trial. After the acoustic signal of the computer, the participants had to push with both legs as strong as possible for 5 seconds against the shin pad. In maximal isokinetic bilateral knee extension and flexion the velocity of isokinetic movement was set at $90 \%$ s. Before the test the load of the legs was annotated and a gravity adjustment was computed using the manufactory software. During this trial, participants had to push and pull the shin pad as fast as possible five times uninterruptedly.

During the two ankle trials, the fulcrum was aligned with the medial malleoli, and the foot was fixed to the support with two stripes. In the maximal isometric ankle plantar and dorsal flexion, the angle was fixed at $30^{\circ}$ of plantar flexion, calculated from the maximal ankle dorsal flexion $\left(0^{\circ}\right)$. After the acoustic signal, the participants had to push down or pull up the ankle as strong possible for 5 seconds, for extension and flexion trial respectively. For the maximal isokinetic ankle plantar flexion and extension, the isokinetic velocity was set to $90 \% \mathrm{sec}$. As for knee, the ankle weight was recorded before the test. Finally, after the acoustic signal the subjects had to push down and pull up the ankle as far possible for five times continuously.

The lengths of the lever arm were recorded and used in the retest trials to avoid position bias.

Before all isokinetic tests, the weight of the legs and the ankles were noted and a gravity adjustment was made using the computer software.

This trial was conducted ethically according to international standards (19).

\section{Data Analysis}

Data were acquired at $1000 \mathrm{~Hz}$. Three trials for each type of test were carried out and maximal torque (in newton-meters) and mean power were recorded. For the analysis, the average of three trials was taken into consideration. Statistical analyses were conducted using SPSS (Version 22.0 for Windows, SPSS Inc., Chicago, IL) and Microsoft Excel 2016. Normal distribution of data was checked using Kolmogorov-Smirnoff (K-S) test. Means and standard deviations were calculated for each variable in both test and retest conditions. In order to examine test-retest reliability the Bland and Altman plots were created (20); limits of agreement (mean of the differences $\pm 1.96 \times \mathrm{SD}$ ) were also reported in the plots. Paired-sample t-tests were performed to detect significant bias between test sessions $(p<0.05)$. Moreover, Intraclass Correlations Coefficients (ICC), 95\% Lower and 95\% Upper Confidential Limits were calculated. Finally, differences between the mean values were also expressed as a percentage in order to allow comparisons among measures and across studies.

\section{RESULTS}

Sample characteristics were reported at Table I. Women participants aged $25.24 \pm 2.56$ years, body mass was 60.76 
$\pm 8.79 \mathrm{~kg}$, height was $1.70 \pm 0.05$ meters, and BMI $20.98 \pm$ 2.47. Men aged $25.61 \pm 2.96$ years, body mass was $74.16 \pm 9.87$ $\mathrm{kg}$, height was $1.81 \pm 0.06 \mathrm{~m}$, and BMI $22.62 \pm 2.38 \mathrm{~kg} / \mathrm{m}^{2}$. All participants concluded the trial safely, any discomfort was indicated.

Paired analyses and Intra-class correlations showed no significant differences $(\mathrm{p}>0.05)$ respectively and an overall good correlation among all assessments. The mean errors (average errors between point estimated) were calculated as the deviations from $0 \mathrm{Nm}$. The Bland and Altman analyses were performed to detect error scores between tests and retest measures. No statistical difference was found between test and retest measures (Table II).

Isometric knee extension showed excellent correlation between test-retest measures (highest 0.96, average 0.95), such as isokinetic knee extension (0.96) and flexion (0.97)

(Figure 2). Isometric right ankle dorsal flexion showed excel- lent correlation (highest 0.96 , average 0.97 ), while plantar flexion showed good correlation for the highest value (0.75) and moderate for the average value (0.71) (Figure 3). For the left ankle isometric dorsal flexion test excellent correlation was found for highest and average results $(0.97$ and $0.98)$, while plantar flexion showed good correlation $(0.78$ and 0.77) (Figure 4). Both right and left isokinetic ankle plantar flexion showed a good correlation (0.88). On the contrary, isokinetic plantar flexion showed good correlation for the left ankle (0.85) and moderate for the right (0.73) (Figure 5). For each test results showed that most values were included within the upper and lower limits of agreement (dashed lines of figures 2-5). The examination of the plots indicates that during maximal isometric right and left dorsal flexion $30^{\circ}$ a tighter distribution was observed when compared with the maximal isometric right and left plantar flexion $30^{\circ}$. The most accurate measure was detected in the

Table II. Means and standard deviations for isometric and isokinetic tests.

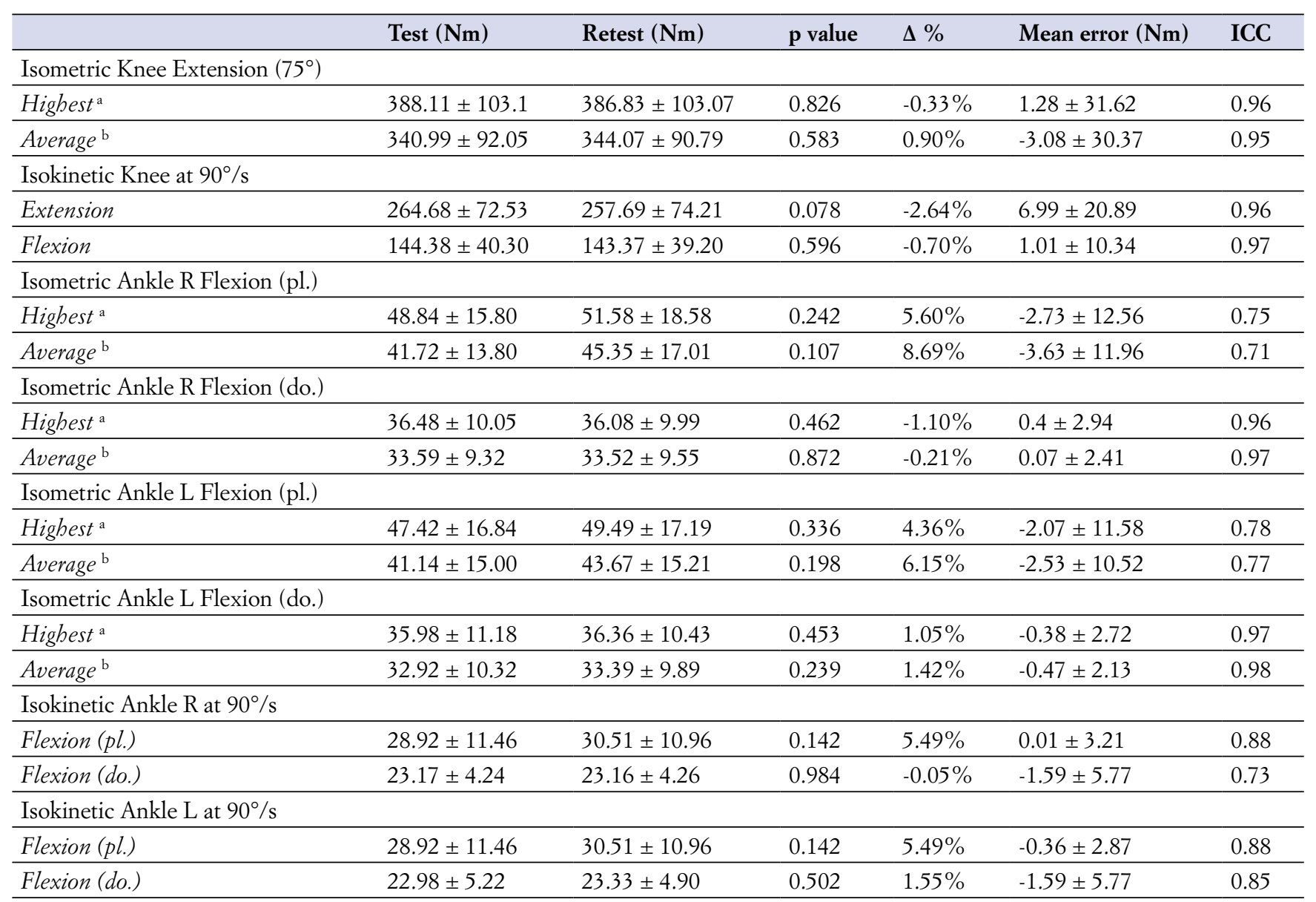

Abbreviation: ICC, Intraclass Correlation Coefficient; ${ }^{\text {a }}$, Highest value of 3 testing procedures; ${ }^{\text {b }}$, Average value of 3 testing procedures; R, right; L, left; *, $p<0.05$; pl., plantar; do., dorsal. 

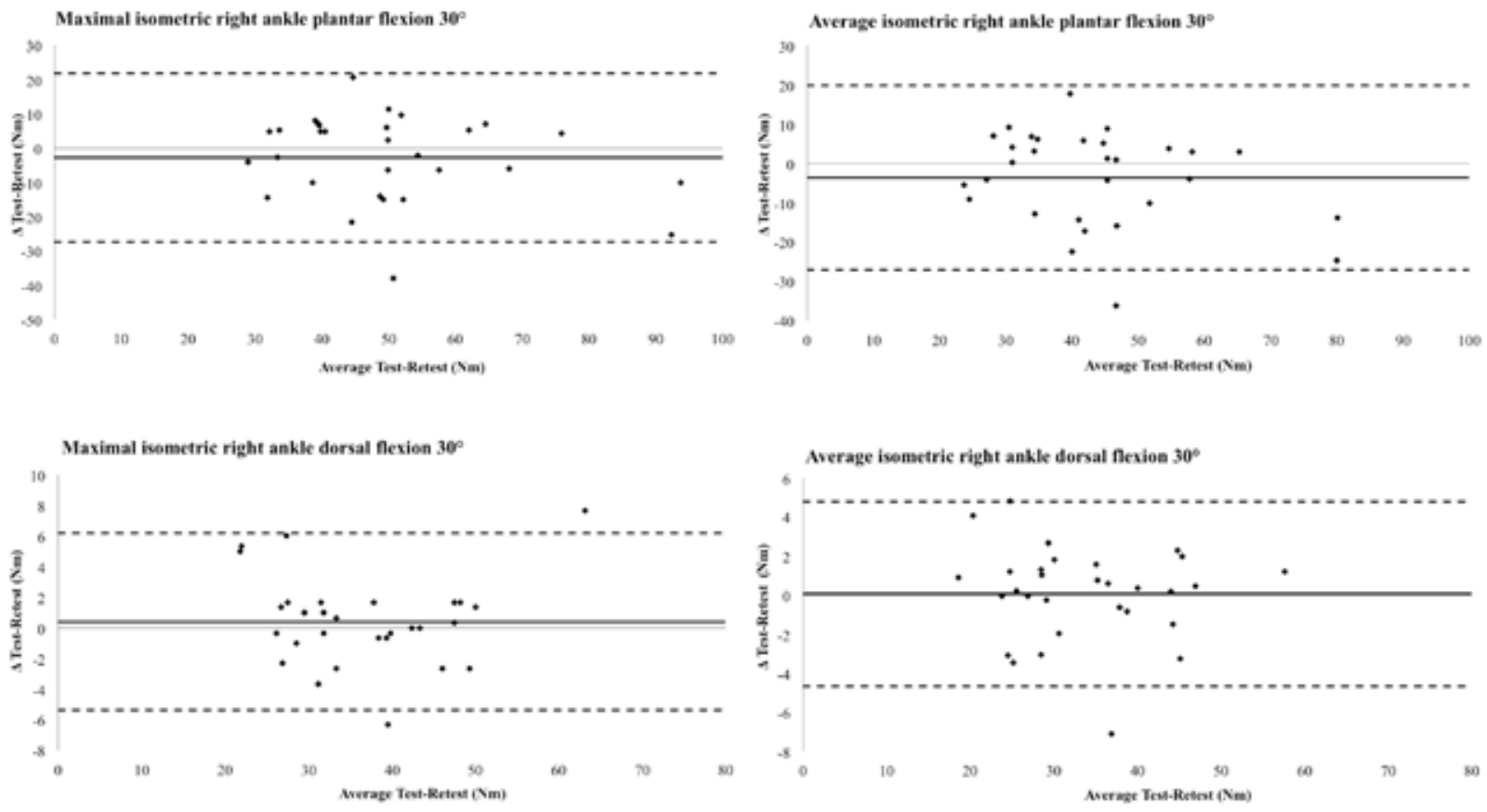

Figure 2. Bland and Altman plots reproducing maximal isometric bilateral knee extension at $75^{\circ}$, average isometric bilateral knee extension at $75^{\circ}$, maximal isokinetic knee extension at $90 \%$ and maximal isokinetic knee flexion at $90 \%$ s.
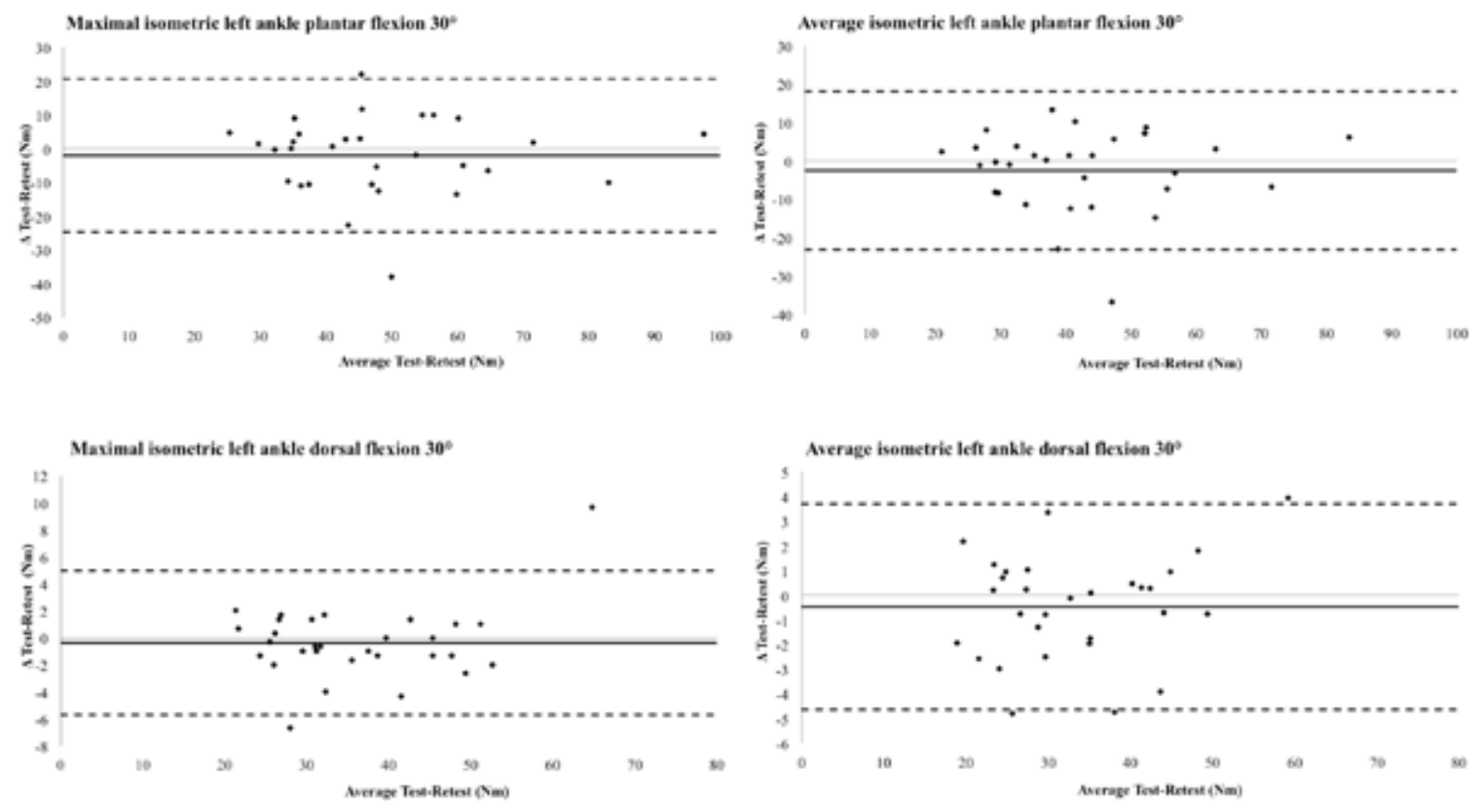

Figure 3. Bland and Altman plots reproducing maximal isometric right ankle plantar flexion at $30^{\circ}$, average isometric right ankle plantar flexion at $30^{\circ}$, maximal isometric right ankle dorsal flexion at $30^{\circ}$ and average isometric right ankle dorsal flexion at $30^{\circ}$. 

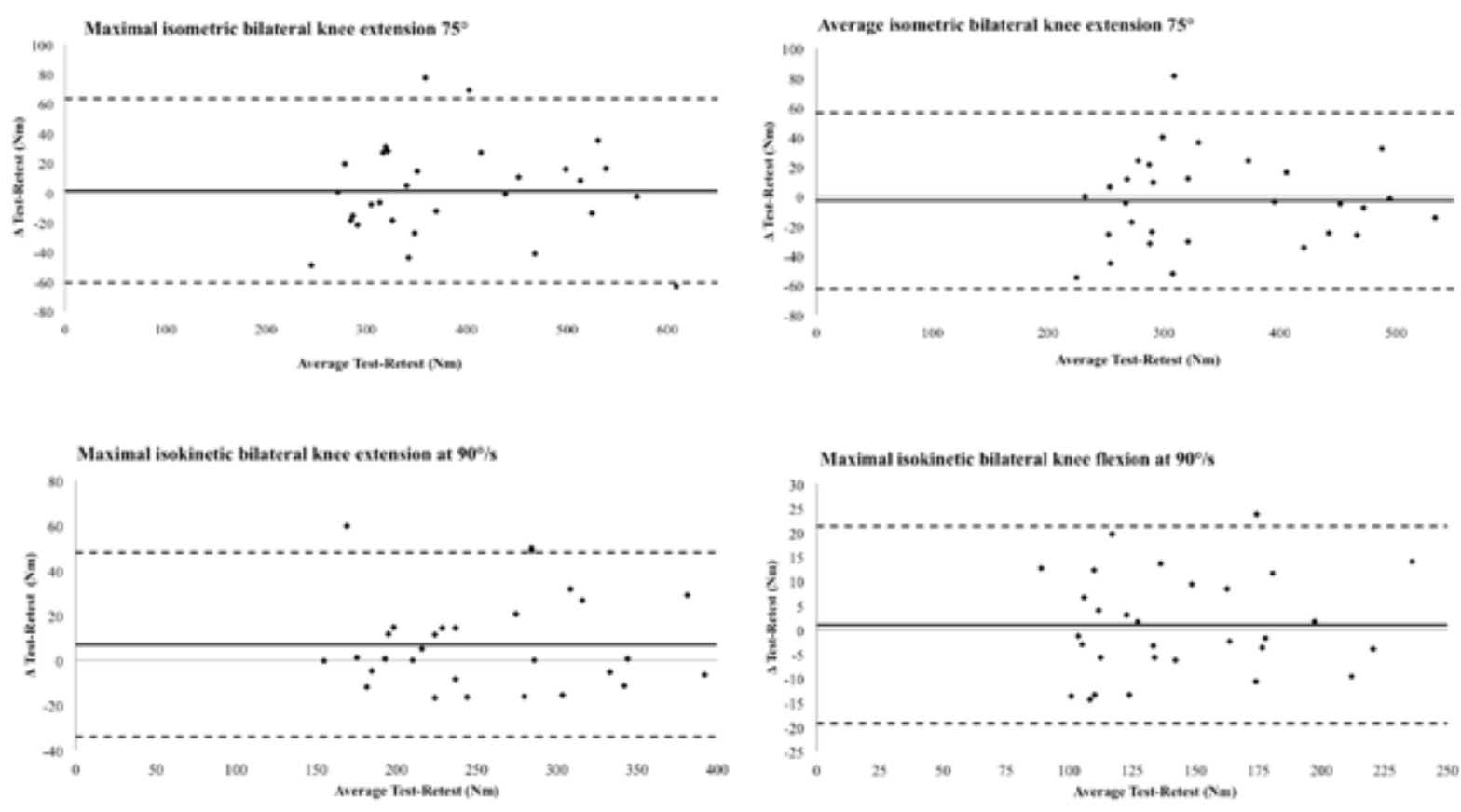

Figure 4. Bland and Altman plots reproducing maximal isometric left ankle plantar flexion at $30^{\circ}$, average isometric left ankle plantar flexion at $30^{\circ}$, maximal isometric left ankle dorsal flexion at $30^{\circ}$ and average isometric left ankle dorsal flexion at $30^{\circ}$.
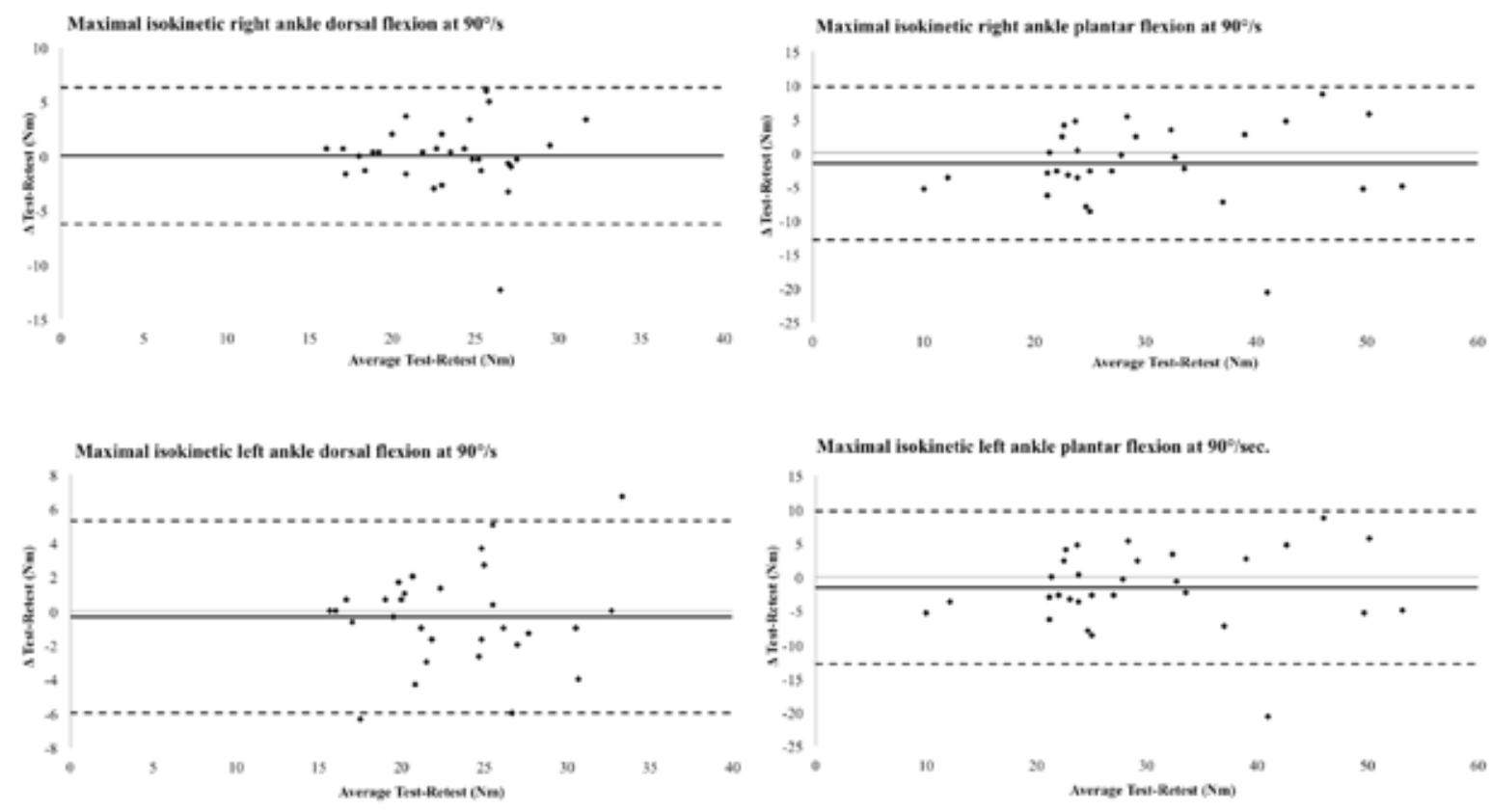

Figure 5. Bland and Altman plots reproducing maximal isokinetic right ankle dorsal flexion at $90 \%$, maximal isokinetic right ankle plantar flexion at $90 \%$, maximal isokinetic left ankle dorsal flexion at $90 \%$ s and maximal isokinetic left ankle plantar flexion at $90 \%$ s. 
maximal isometric bilateral knee extension test at $75^{\circ}$, while the worst was in the maximal isometric right ankle plantar flexion at $30^{\circ}$ test.

\section{DISCUSSION}

The aim of this study is to observe the test-retest reliability of knee and ankle isokinetic and isometric strength measures using the isokinetic dynamometer provided by Easytech in healthy young adults. Overall, the results reveal a very high reliability. With more details, in isometric knee extension, isokinetic knee extension at $90 \%$, isometric right ankle dorsal flexion and isometric left ankle dorsal flexion, ICC is higher than 0.90 . Moreover, in all the other tests the ICC is higher than 0.70 meaning a good correlation between the test and retest measures. Additionally, observing the Bland and Altman plots, the prevalence of values was placed within the limits of agreements and the correspondence between test and retest differences confirms the reproducibility of the assessments executed with the multi-joint evaluation system Prima Plus (Easytech, Italy). Besides, the percentage differences between test and retest measures were similar to previous studies involving young subjects in a test-retest reliability of isokinetic knee extension and flexion (1).

Analyzing data from the differences between test and retest, some similarities can be detected between right and left ankle both on isokinetic and isometric testing trials. There is an analogous percentage difference and ICC between the isometric right $(5.60 \%, 0.75)$ and left $(4.36 \%, 0.78)$ ankle plantar flexion, between isometric right $(-1.10 \%, 0.96)$ and left $(1.05 \%, 0.97)$ ankle dorsal flexion and also between isokinetic right and left plantar $(5.49 \%, 0.88 ; 5.49 \%, 0.88)$ and dorsal $(-0.05 \%, 0.73 ; 1.55 \%, 0.85)$ flexion. These results indicated a high reliability of the device but also that the strength of the analyzed sample was apparently well balanced between right and left ankle even though the large majority of participants was right handed and the dominant leg was the right.

Another notable result was that the most accurate measure was detected in the maximal isometric bilateral knee extension test at $75^{\circ}$. This could be due to the fact that some participants had previous experiences with devices alike leg

\section{REFERENCES}

1. Sole G, Hamren J, Milosavljevic S, Nicholson H, Sullivan SJ. Test-retest reliability of isokinetic knee extension and flexion. Arch Phys Med Rehabil. 2007;88(5):626-31.

2. Drouin JM, Valovich-mcLeod TC, Shultz SJ, Gansneder BM, Perrin DH. Reliability and validity of the Biodex system 3 pro isokinetic dynamometer velocity, torque and position measurements. Eur J Appl Physiol. 2004;91(1):22-9. extension despite no one reported previous experience with isometric and isokinetic muscular strength test.

Furthermore, despite the examined sample was not extensively large, participants involved in our study could be considered as representative of an average healthy young adults' population, in fact their activity level varied from sedentary to regular exerciser behavior, representing a heterogeneous group and wide range of strength values. Moreover, sample size was larger than previous studies analyzing reliability of isometric and isokinetic leg extension and flexion in healthy young adults $(1,15)$. To minimize sources of errors, testing procedure and instructions were standardized. Seat coordinates and machine arm length were recorded as indicator of individual position. Randomization of testing order ensured absence of interaction effect. The use of only one isokinetic testing velocity $\left(90^{\circ} / \mathrm{s}\right)$ could be considered the main limitations for this study that could limit peak torque. Anyway, participants had no experience in isokinetic strength testing procedure, and different speed could affect their ability to end sessions.

Considering the variety of tests that can be administered with the device object of this investigation, future analyses involving either athletes and patients should be an opportunity of study also using different angular velocities, for example $60 \%$, for isokinetic test or different angle of extension for isometric test.

\section{CONCLUSION}

The results of this study verified a high reliability of the multi-joint evaluation system Prima Plus in the assessment of knee and ankle isokinetic and isometric strength among healthy young adults. The evaluation of knee muscle imbalances or bilateral asymmetry between the two ankles could be applied for exercise prescription in healthy adults to reduce the risk of injuries during physical activity, especially in sedentary subjects that just started to exercise.

\section{Conflict of interests}

The authors declare no conflict of interest. 
5. Croisier JL. Factors associated with recurrent hamstring injuries. Sports Med. 2004;34(10):681-95.

6. Perrin DH. Isokinetic Exercise and Assessment: Human Kinetics Publishers; 1993.

7. de Araujo Ribeiro Alvares JB, Rodrigues R, de Azevedo Franke $\mathrm{R}$, da Silva BG, Pinto RS, Vaz MA, et al. Inter-machine reliability of the Biodex and Cybex isokinetic dynamometers for knee flexor/extensor isometric, concentric and eccentric tests. Phys Ther Sport. 2015;16(1):59-65.

8. Almosnino S, Stevenson JM, Bardana DD, Diaconescu ED, Dvir Z. Reproducibility of isokinetic knee eccentric and concentric strength indices in asymptomatic young adults. Phys Ther Sport. 2012;13(3):156-62.

9. Hartmann A, Knols R, Murer K, de Bruin ED. Reproducibility of an isokinetic strength-testing protocol of the knee and ankle in older adults. Gerontology. 2009;55(3):259-68.

10. de Carvalho Froufe Andrade AC, Caserotti P, de Carvalho CM, de Azevedo Abade EA, da Eira Sampaio AJ. Reliability of Concentric, Eccentric and Isometric Knee Extension and Flexion when using the REV9000 Isokinetic Dynamometer. J Hum Kinet. 2013;37:47-53.

11. Impellizzeri FM, Bizzini M, Rampinini E, Cereda F, Maffiuletti NA. Reliability of isokinetic strength imbalance ratios measured using the Cybex NORM dynamometer. Clin Physiol Funct Imaging. 2008;28(2):113-9.

12. Orri JC, Darden GF. Technical report: Reliability and validity of the iSAM 9000 isokinetic dynamometer. J Strength Cond Res. 2008;22(1):310-7.
13. Fagher K, Fritzson A, Drake AM. Test-Retest Reliability of Isokinetic Knee Strength Measurements in Children Aged 8 to 10 Years. Sports Health. 2016;8(3):255-9.

14. Bergamin M, Gobbo S, Bullo V, Vendramin B, Duregon F, Frizziero A, et al. Reliability of a device for the knee and ankle isometric and isokinetic strength testing in older adults. Muscles Ligaments Tendons J. 2017;7(2):323-30.

15. Parsons JL, Porter MM. Reliability of measuring hip and knee power and movement velocity in active youth. Pediatr Phys Ther. 2015;27(1):82-9.

16. Folstein MF, Folstein SE, McHugh PR. "Mini-mental state”. A practical method for grading the cognitive state of patients for the clinician. J Psychiatr Res. 1975;12(3):189-98.

17. Tombaugh TN, McIntyre NJ. The mini-mental state examination: a comprehensive review. J Am Geriatr Soc. 1992;40(9):922-35.

18. Larsson B, Karlsson S, Eriksson M, Gerdle B. Test-retest reliability of EMG and peak torque during repetitive maximum concentric knee extensions. J Electromyogr Kinesiol. 2003;13(3):281-7.

19. Padulo J. OF, Frizziero A., Maffulli N. Muscles, Ligaments and Tendons Journal - Basic principles and recommendations in clinical and field Science Research: 2018 update. MLTJ. 2018;8(3):305-7.

20. Bland JM, Altman DG. Statistical methods for assessing agreement between two methods of clinical measurement. Lancet. 1986;1(8476):307-10. 